\title{
On a Berry-Esseen type limit theorem for Boolean convolution*
}

\author{
Mauricio Salazar ${ }^{\dagger}$
}

\begin{abstract}
We obtain a sharp estimate of the speed of convergence in the Boolean central limit theorem for measures with finite sixth moment. The main tool is a quantitative version of the Stieltjes-Perron inversion formula.
\end{abstract}

Keywords: Stieltjes-Perron formula; Lévy distance; Boolean central limit theorem; Berry-Esseen theorem.

MSC2020 subject classifications: $46 \mathrm{~L} 53$.

Submitted to ECP on November 4, 2020, final version accepted on January 10, 2022.

\section{Introduction}

In Non-commutative probability, the concept of random variable is generalized to a scheme of operators where new notions of independence of random variables appear. Some of the most important notions of independence [10] are the free, Boolean, and monotone ones.

One of the main objectives in non-commutative probability is to obtain analogues of relevant theorems in classical probability. Such is the case of the Berry-Esseen theorem $[4,6]$. This theorem is a quantitative version of the Central Limit Theorem (CLT) that is important to justify many applications. Recall that the Kolmogorov distance between two probability measures $\mu$ and $\nu$ is defined by $d_{k o l}(\mu, \nu):=\sup _{x \in \mathbb{R}}|\mu((-\infty, x])-\nu((-\infty, x])|$. Let $m_{n}(\mu)$ denote the $\mathrm{n}$-th moment of a measure $\mu$. The Berry-Esseen theorem states that if $\mu$ is a probability measure with $m_{1}(\mu)=0, m_{2}(\mu)=1$, and $\int_{\mathbb{R}}|t|^{3} d \mu(t)=\rho<\infty$, then there exist an absolute constant $C>0$ such that

$$
d_{\text {kol }}\left(D_{\frac{1}{\sqrt{n}}} \mu^{* n}, \mathcal{N}\right) \leq C \rho n^{-1 / 2},
$$

where $*$ stands for the convolution, $\mathcal{N}$ represents the standard normal distribution, and $D_{a} \mu$ denotes the dilation of a measure $\mu$ by a factor $a>0$; this means that $D_{a} \mu(B)=$ $\mu\left(a^{-1} B\right)$ for all Borel sets $B \subset \mathbb{R}$.

The proof of the Berry-Esseen theorem, see Section XVI.5 in Feller [7], is based on a general smoothing inequality in terms of the Fourier transform to bound the Kolmogorov distance between distributions and on obtaining an adequate approximation to the Fourier transform of the n-fold iteration of the classic convolution.

\footnotetext{
*The author thanks the support of the PRODEP postdoc program of the UASLP

${ }^{\dagger}$ Institito de Física, UASLP. E-mail: maurma@cimat . mx
} 
In free probability, the analogue of the Berry-Esseen theorem for the free CLT was obtained by Chistyakov and Götze [5]. Their result says that if $\mu$ is a measure with $m_{1}(\mu)=0, m_{2}(\mu)=1$, and $m_{4}(\mu)<\infty$, then there exist an absolute constant $C>0$ such that

$$
d_{k o l}\left(D_{\frac{1}{\sqrt{n}}} \mu^{\boxplus n}, S\right) \leq C\left(\left|m_{3}(\mu)\right|+m_{4}(\mu)^{1 / 2}\right) n^{-1 / 2},
$$

where $\boxplus$ stands for the free convolution and $S$ represents the standard semicircle distribution.

In monotone probability theory, Wang, Arizmendi, and Salazar [2] proved a BerryEsseen type estimate for the monotone CLT: if $\mu$ is a measure with $m_{1}(\mu)=0, m_{2}(\mu)=1$, and finite sixth moment, then $d_{k o l}\left(D_{\frac{1}{\sqrt{n}}} \mu^{\triangleright n}, \mathbf{a}\right)=O\left(n^{-1 / 4}\right)$, where $\triangleright$ stands for the monotone convolution and a denotes the standard arcsine distribution.

The proofs in [5] and [2] to obtain the Berry-Esseen type estimates for the central limit theorems in free and monotone probability, respectively, rely on Bai's inequality [3], which is a smoothing inequality in terms of the Cauchy transform (see Subsection 2.2) to bound the Kolmogorov distance between two distributions, and on obtaining adequate expressions for the Cauchy transform of the $\mathrm{n}$-fold iteration of the free and monotone convolutions.

In the case of Boolean probability, a Berry-Essen type result for the Boolean CLT was obtained by Arizmendi and Salazar [1]. In this case, convergence in the Kolmogorov distance does not hold. Instead, one should use Levy distance, denoted by $d_{l e v}$ (see Subsection 2.1), since it metrizes weak convergence of measures. Let $\uplus$ denote the Boolean convolution and $\mathbf{b}$ denote the symmetric Bernoulli distribution, $\frac{1}{2} \delta_{-1}+\frac{1}{2} \delta_{1}$. Suppose that $\mu$ is a probability measure with $m_{1}(\mu)=0$ and $m_{2}(\mu)=1$. Arizmendi and Salazar proved that if $\mu$ has bounded support, then $d_{l e v}\left(D_{\frac{1}{\sqrt{n}}} \mu^{\uplus n}, \mathbf{b}\right)=O\left(n^{-1 / 2}\right)$, and if $\mu$ has finite fourth moment, then $d_{l e v}\left(D_{\frac{1}{\sqrt{n}}} \mu^{\uplus n}, \mathbf{b}\right)=O\left(n^{-1 / 3}\right)$.

In this paper, we obtain the following theorem that improves the above results on Berry-Esseen type estimates for the Boolean CLT. The estimate in this theorem is sharp, see Remark 3.1.

Theorem 1.1. Let $\mu$ be a probability measure such that $m_{1}(\mu)=0, m_{2}(\mu)=1$, and $m_{6}(\mu)<\infty$. Define the measure $\mu_{n}:=D_{\frac{1}{\sqrt{n}}} \mu^{\uplus n}$. Then, for $n$ large enough we have that

$$
d_{l e v}\left(\mu_{n}, \mathbf{b}\right) \leq \frac{7}{2} \frac{C+2}{\sqrt{n}}
$$

where $C$ is a constant that depends only on $\mu$.

In the bounded case, the proof in [1] of the Berry-Esseen type estimate for the Boolean CLT is based on accurately approximating the Cauchy transform of the $n$-fold iteration of the Boolean convolution, while in the unbounded case the proof is based on a general inequality to bound the Levy distance of any measure to the Bernoulli distribution.

Now, the proof of Theorem 1 is also based on such inequality. However, we combine it with a quantitative version of the Stieltjes-Perron inversion formula (Theorem 2 below) and further refinements of estimates related to the Cauchy transform.

Apart from this introduction, the sections of this paper are organized as follows. In Section 2, we present the preliminary material and technical results necessary to prove our main result. Particularly, in Section 2.2, we present Theorem 2 and discuss some interesting consequences. In Section 3, we prove Theorems 1 and 2. 


\section{Preliminaries}

In this section we define all the concepts used in this work and discuss some basic properties of them. In Subsection 2.1, we define the Levy distance and state a proposition that gives an upper bound of the Levy distance of a probability measure to the Bernoulli measure. In Subsection 2.2, we introduce the Cauchy transform and the F-transform. We also state Theorem 2 which gives a quantitative estimate to recover a measure from its Cauchy transform. In Subsection 2.3, we define the Boolean convolution in terms of the F-transform and obtain an expression for the F-transform of the n-fold iteration of the Boolean convolution of a measure with itself.

\subsection{The Lévy distance}

Let $\mu$ and $\nu$ probability measures. We define the Lévy distance, see [8], between them to be

$$
d_{l e v}(\mu, \nu):=\inf \{\epsilon>0 \mid F(x-\epsilon)-\epsilon \leq G(x) \leq F(x+\epsilon)+\epsilon \text { for all } x \in \mathbb{R}\},
$$

where $F$ and $G$ are the cumulative distribution functions of $\mu$ and $\nu$ respectively.

The following Proposition is the Lemma 2 in [1], and it is a key ingredient in the proof of Theorem 1.

Proposition 2.1. Let $\mu$ be a probability measure of zero mean and unit variance. Suppose further that $\mu((-1-\epsilon,-1+\epsilon) \cup(1-\epsilon, 1+\epsilon)) \geq 1-\epsilon$ for some $\epsilon \in(0,1)$. Then

$$
d_{l e v}(\mu, \mathbf{b}) \leq \frac{7}{2} \epsilon .
$$

\subsection{The Cauchy transform}

Throughout the paper $z$ denotes a complex number and we write $z=x+i y$, where $x$ and $y$ are real numbers.

The Cauchy transform (or Stieltjes transform) [12] of a non-negative Borel measure $\mu$ is defined as

$$
G_{\mu}(z):=\int_{\mathbb{R}} \frac{1}{z-t} d \mu(t) \text { for } z \in \mathbb{C}^{+},
$$

where $\mathbb{C}^{+}$denotes the open upper complex half-plane.

We can recover a measure $\mu \in \mathcal{M}$ from its Cauchy transform via the Stieltjes-Perron inversion formula:

$$
\mu([a, b])=\lim _{y \downarrow 0}-\frac{1}{\pi} \int_{a}^{b} \Im\left(G_{\mu}(x+i y)\right) d x,
$$

provided that $\mu(\{a, b\})=0$.

The following theorem is a quantitative version of the Stieltjes-Perron inversion formula which is tailored for our purposes. This will be proved in Section 3.1.

Theorem 2.2. Let $\mu$ be a probability measure. Let $-\infty \leq a<b \leq \infty$. Then we have that for all $y>0$

$$
\mu((a+\delta, b-\delta])-\frac{2 y}{\pi \delta} \leq-\frac{1}{\pi} \int_{a}^{b} \Im G_{\mu}(x+i y) d x \leq \mu((a-\delta, b+\delta])+\frac{2 y}{\pi \delta} .
$$

Note that if $\delta=\sqrt{y}$ and $y \downarrow 0$, then we obtain the Stieltjes-Perron inversion formula for probability measures. Moreover, taking $a=-\infty$ and $\delta=\sqrt{\frac{2 y}{\pi}}$, we deduce that

$$
d_{l e v}\left(\mu^{y}, \mu\right) \leq \sqrt{\frac{2 y}{\pi}}
$$


where $\mu^{y}$ is the probability measure of density $-\frac{1}{\pi} \Im\left(G_{\mu}(x+i y)\right) d x$, i.e. $\mu^{y}=\mu * C_{y}$ where $C_{y}$ is the Cauchy distribution with location 0 and scale $y$. This further implies the following inequality for the Lévy distance between two probability measures in terms of the Cauchy transform

$$
d_{l e v}(\mu, \nu) \leq \sqrt{\frac{8 y}{\pi}}+\frac{1}{\pi} \int_{\mathbb{R}}\left|\Im\left(G_{\mu}(z)\right)-\Im\left(G_{\nu}(z)\right)\right| d x .
$$

Next, we discuss some bounds for the Cauchy transform.

Since $|z-t| \geq y$ for all $t \in \mathbb{R}$, then it follows that

$$
\left|G_{\mu}(z)\right| \leq \frac{\mu(\mathbb{R})}{y} \text { for } z \in \mathbb{C}^{+}
$$

The following proposition gives another bound for $\left|G_{\mu}(z)\right|$ in terms of the moments of $\mu$.

Proposition 2.3. Let $\mu$ be a measure and $i \geq 0$. Then we have that

$$
\left|G_{\mu}(z)\right|<\frac{2 \mu(\mathbb{R})}{|x|}+\frac{2^{i} \int_{\mathbb{R}}|t|^{i} d \mu(t)}{y|x|^{i}}
$$

for any $x>0$.

Proof. We have that

$$
\begin{aligned}
\left|G_{\mu}(z)\right| & \leq \int_{|t| \leq \frac{|x|}{2}} \frac{1}{|x-t|} d \mu(t)+\int_{|t|>\frac{|x|}{2}} \frac{1}{y} d \mu(t) \\
& \leq \frac{2}{|x|} \int d \mu(t)+\frac{1}{y} \int_{|t|>\frac{|x|}{2}} d \mu(t) \\
& <\frac{2 \mu(\mathbb{R})}{|x|}+\frac{2^{i} \int_{\mathbb{R}}|t|^{i} d \mu(t)}{y|x|^{i}}
\end{aligned}
$$

In particular, taking $i=2$ we obtain that

$$
\left|G_{\mu}(z)\right|<\frac{2 \mu(\mathbb{R})}{|x|}+\frac{4 \int t^{2} d \mu(t)}{y x^{2}} .
$$

The reciprocal Cauchy transform (or $F$-transform) of a positive Borel measure $\mu \in \mathcal{M}$ is defined as

$$
F_{\mu}(z):=\frac{1}{G_{\mu}(z)} \quad \text { for } z \in \mathbb{C}^{+} .
$$

Directly by definition, it is not too difficult to see that for a probability measure $\mu$ and $a>0$, then

$$
F_{D_{a} \mu}(z)=a F_{\mu}(z / a) \quad \text { for } z \in \mathbb{C}^{+} .
$$

The next proposition gives a fundamental representation of the $F$-transform for the probability measures that are of our interest.

Proposition 2.4. Let $\mu$ a probability measure such that $m_{1}(\mu)=0, m_{2}(\mu)=1$, and $m_{6}(\mu)<\infty$. Then there exists a real number $\alpha$ and a non-negative Borel measure $\omega$ such that $m_{2}(\omega)<\infty$ and

$$
F_{\mu}(z)=z-\frac{1}{z-\alpha-G_{\omega}(z)} \quad \text { for } z \in \mathbb{C}^{+}
$$


Proof. Let $\mu$ as in the hypothesis. Since $m_{1}(\mu)=0$ and $m_{2}(\mu)=1$, then by Proposition 2.1 in [9] we have that there exists a probability measure $\nu$ such that

$$
F_{\mu}(z)=z-G_{\nu}(z) \text { for } z \in \mathbb{C}^{+}
$$

Moreover, since $m_{6}(\mu)<\infty$, then by Proposition 4.8 also in [9] we have that $m_{4}(\nu)<$ $\infty$. Again by the same propositions aplied to $\nu$, we get that there exists a real number $\alpha$ and non-negative measure $\omega$ such that $m_{2}(\omega)<\infty$ and

$$
F_{\nu}(z)=z-\alpha-G_{\omega}(z) \text { for } z \in \mathbb{C}^{+} \text {. }
$$

The desired representation follows from the above equations.

\subsection{Boolean convolution}

Given probability measures $\mu$ and $\nu$, the Boolean convolution $\mu \uplus \nu$, introduced by Speicher and Woroudi [11], is the probability measure defined by the equation

$$
F_{\mu \uplus \nu}(z)=F_{\mu}(z)+F_{\nu}(z)-z \text { for } z \in \mathbb{C}^{+} .
$$

Let $\mu$ be a probability measure and $n$ be a positive integer. We want to obtain an expression for the F-transform of $\mu_{n}:=D_{\frac{1}{\sqrt{n}}}\left(\mu^{\uplus n}\right)$.

First note that

$$
F_{\mu^{\uplus n}}(z)=(1-n) z+n F_{\mu}(z) \text { for } z \in \mathbb{C}^{+} .
$$

Now, suppose further that $m_{1}(\mu)=0, m_{2}(\mu)=1$, and $m_{6}(\mu)<\infty$. Thus, by Proposition 2.4 we get that

$$
F_{\mu^{\uplus n}}(z)=z-\frac{n}{z-\alpha-G_{\omega}(z)} \text { for } z \in \mathbb{C}^{+},
$$

where $\alpha$ is a real number and and $\omega$ is a non-negative Borel measure such that $\int t^{2} d \omega(t)<$ $\infty$. Finally, applying (2.3) we obtain the representation

$$
F_{\mu_{n}}(z)=z-\frac{1}{z-\frac{\alpha}{\sqrt{n}}-\frac{1}{\sqrt{n}} G_{\omega}(\sqrt{n} z)} \quad \text { for } z \in \mathbb{C}^{+}
$$

\section{Proofs}

\subsection{Proof of Theorem 2.2.}

Let $\mu$ be a probability measure. Choose $a$ and $b$ such that $-\infty \leq a<b \leq \infty$ and fix $y>0$. First, we rewrite the following integral

$$
\begin{aligned}
\int_{a}^{b}-\Im G_{\mu}(x+i y) d x & =\int_{a}^{b} \int_{-\infty}^{\infty} \frac{y}{(x-t)^{2}+y^{2}} d \mu(t) d x \\
& =\int_{-\infty}^{\infty} \int_{a}^{b} \frac{y}{(x-t)^{2}+y^{2}} d x d \mu(t) \\
& =\int_{-\infty}^{\infty} \int_{\frac{a-t}{y}}^{\frac{b-t}{y}} \frac{1}{x^{2}+1} d x d \mu(t)
\end{aligned}
$$

Now, let $\delta \in\left(0, \frac{b-a}{2}\right)$. It follows that 
On a Berry-Esseen type limit theorem for Boolean convolution

$$
\begin{aligned}
\int_{-\infty}^{\infty} \int_{\frac{a-t}{y}}^{\frac{b-t}{y}} \frac{1}{x^{2}+1} d x d \mu(t) & \geq \int_{a+\delta}^{b-\delta} \int_{\frac{a-t}{y}}^{\frac{b-t}{y}} \frac{1}{x^{2}+1} d x d \mu(t) \\
& \geq \int_{a+\delta}^{b-\delta} \int_{\frac{-\delta}{y}}^{\frac{\delta}{y}} \frac{1}{x^{2}+1} d x d \mu(t) \\
& =\int_{a+\delta}^{b-\delta}\left(\pi-\int_{|t|>\frac{\delta}{y}} \frac{1}{x^{2}+1} d x\right) d \mu(t) \\
& \geq \int_{a+\delta}^{b-\delta}\left(\pi-\frac{2 y}{\delta}\right) d \mu(t) \\
& \geq \pi \mu((a+\delta, b-\delta])-\frac{2 y}{\delta}
\end{aligned}
$$

So, we arrive to

$$
\mu((a+\delta, b-\delta])-\frac{2 y}{\pi \delta} \leq-\frac{1}{\pi} \int_{a}^{b} \Im G_{\mu}(x+i y) d x .
$$

On the other hand, we have that

$$
\int_{a}^{b}-\Im G_{\mu}(x+i y) d x=\int_{a-\delta}^{b+\delta} \int_{\frac{a-t}{y}}^{\frac{b-t}{y}} \frac{1}{x^{2}+1} d x d \mu(t)+\int_{t \notin(a-\delta, b+\delta]} \int_{\frac{a-t}{y}}^{\frac{b-t}{y}} \frac{1}{x^{2}+1} d x d \mu(t) .
$$

Next, note that

$$
\int_{a-\delta}^{b+\delta} \int_{\frac{a-t}{y}}^{\frac{b-t}{y}} \frac{1}{x^{2}+1} d x d \mu(t) \leq \int_{a-\delta}^{b+\delta} \int_{-\infty}^{\infty} \frac{1}{x^{2}+1} d x d \mu(t)=\pi \mu((a-\delta, b+\delta]) .
$$

Hence, splitting the integral over the complement of the interval $(a-\delta, b+\delta]$, we get

$$
\begin{aligned}
\int_{t \notin(a-\delta, b+\delta]} \int_{\frac{a-t}{y}}^{\frac{b-t}{y}} \frac{1}{x^{2}+1} d x d \mu(t) & =\int_{-\infty}^{a-\delta} \int_{\frac{a-t}{y}}^{\frac{b-t}{y}} \frac{1}{x^{2}+1} d x d \mu(t)+\int_{b+\delta}^{\infty} \int_{\frac{a-t}{y}}^{\frac{b-t}{y}} \frac{1}{x^{2}+1} d x d \mu(t) \\
& \leq \int_{-\infty}^{a-\delta} \int_{\frac{\delta}{y}}^{\infty} \frac{1}{x^{2}+1} d x d \mu(t)+\int_{b+\delta}^{\infty} \int_{-\infty}^{\frac{-\delta}{y}} \frac{1}{x^{2}+1} d x d \mu(t) \\
& \leq 2 \int_{\frac{\delta}{y}}^{\infty} \frac{1}{x^{2}+1} d x d \mu(t) \\
& =\frac{2 y}{\delta} .
\end{aligned}
$$

Finally, we conclude that for any $y>0$

$$
\frac{-1}{\pi} \int_{a}^{b} \Im G_{\mu}(x+i y) d x \leq \mu((a-\delta, b+\delta])+\frac{2 y}{\pi \delta} .
$$

\subsection{Proof of Theorem 1.1}

We are now ready to prove Theorem 1. Before this, let us make the following remark. Remark 3.1. In [1] it is also shown that if we define $p_{n}:=\frac{1}{2} \frac{\sqrt{1+4 n}+1}{\sqrt{1+4 n}}, q_{n}:=\frac{1}{2} \frac{\sqrt{1+4 n}-1}{\sqrt{1+4 n}}$, $x_{n}:=\frac{1-\sqrt{1+4 n}}{\sqrt{4 n}}$, and $y_{n}:=\frac{1+\sqrt{1+4 n}}{\sqrt{4 n}}$, then the probability measure given by $\mu_{n}:=p_{n} \delta_{x_{n}}+$ $q_{n} \delta_{y_{n}}$ is such that $\mu_{n}=D_{\frac{1}{\sqrt{n}}} \mu_{1}^{\uplus n}$ and $L\left(\mu_{n}, \mathbf{b}\right) \geq \frac{1}{6 \sqrt{n}}$. 
Now, let us fix a probability measure $\mu$ such that $m_{1}(\mu)=0, m_{2}(\mu)=1$, and $m_{6}(\mu)<$ $\infty$. Define $\mu_{n}:=D_{\frac{1}{\sqrt{n}}}\left(\mu^{\uplus n}\right)$. We begin by obtaining some representations for the imaginary part of the Cauchy transform of $\mu_{n}$.

Note that

$$
-\Im\left(G_{\mu_{n}}(z)\right)=\frac{\Im\left(F_{\mu_{n}}(Z)\right)}{\left|F_{\mu_{n}}(z)\right|^{2}} .
$$

Recall that by (2.4) we have the representation

$$
F_{\mu_{n}}(z)=z-\frac{1}{z-\frac{\alpha}{\sqrt{n}}-\frac{1}{\sqrt{n}} G_{\omega}(\sqrt{n} z)} \text { for } z \in \mathbb{C}^{+},
$$

where $\alpha$ is a real number and $\omega$ is a non-negative Borel measure such that $\int t^{2} d \omega(t)<\infty$. Define $W_{n}(z)=z-\frac{\alpha}{\sqrt{n}}-\frac{1}{\sqrt{n}} G_{\omega}(\sqrt{n} z)$ so that $F_{\mu_{n}}(z)=z-\frac{1}{W_{n}(Z)}$. It follows that

$$
-\Im G_{\mu_{n}}(z)=\frac{y\left|W_{n}(z)\right|^{2}+\Im\left(W_{n}(z)\right)}{\left|z W_{n}(z)-1\right|^{2}} .
$$

Next, we establish two lemmas that carry the main estimations of the proof. But first, we define some constants and give an inequality that is vital for making such estimations. Let $K=\max \left\{\omega(\mathbb{R}), \int t^{2} d \omega(t)\right\}$. Now, take $C>\max \left\{5,|\alpha|+2,4(K+1)^{2}, 1+\frac{1}{0.3^{2}}(30 K+1)\right\}$, let $n>\max \left\{20^{2} \alpha^{2}, 20 \cdot 30 K, 16 C^{2}\right\}$, and fix $y=\frac{1}{n}$. Observe that by the inequality (2.2) we deduce that

$$
\left|G_{\omega}(\sqrt{n} z)\right|<\frac{2 K}{\sqrt{n}|x|}+\frac{4 K}{\sqrt{n} x^{2}} .
$$

Lemma 3.2. We have that $-\frac{1}{\pi} \int_{A_{i}} \Im G_{\mu_{n}}(z) d x \leq \frac{1}{\pi \sqrt{n}}$ for $i=1,2$, provided that $n$ is large enough, and where $A_{1}=\left(-\infty,-1-\frac{C}{\sqrt{n}}\right]$ and $A_{2}=\left[1+\frac{C}{\sqrt{n}}, \infty\right)$.

Proof. Assume that $x \leq-1-\frac{C}{\sqrt{n}}$. Since $|x|>1$, then by (3.3) we have that $\left|G_{\omega}(\sqrt{n} z)\right|<$ $\frac{6 K}{\sqrt{n}}$.

First, we want to bound below $\left|F_{\mu_{n}}(z)\right|$. Observe that $\Re\left(W_{n}(z)\right) \leq x+\frac{|\alpha|}{\sqrt{n}}+\frac{1}{\sqrt{n}}\left|G_{\omega}(\sqrt{n} z)\right|$ $<x+\frac{|\alpha|}{\sqrt{n}}+\frac{6 K}{n}$. As $x \leq-1-\frac{C}{\sqrt{n}}, \sqrt{n}>6 K$, and $C>|\alpha|+1$, then it follows that $\Re\left(W_{n}(z)\right)<-1$. Therefore, $\left|W_{n}(z)\right|>1$, which further implies $\left|\frac{1}{W_{n}(z)}\right|<1$. Hence, we deduce that $\left|\Re\left(\frac{1}{W_{n}(z)}\right)\right|<1$. Using this, we conclude that

$$
\left|\Re\left(F_{\mu_{n}}(z)\right)\right|=\left|x-\Re\left(\frac{1}{W_{n}(z)}\right)\right| \geq|x|-\left|\Re\left(\frac{1}{W_{n}(z)}\right)\right|>-x-1,
$$

for $x \leq-1-\frac{C}{\sqrt{n}}$.

Now, we want to bound above $\Im\left(F_{\mu_{n}}(z)\right)$. As seen above, for $x \leq-1-\frac{C}{\sqrt{n}}$, one has that $-\Im\left(G_{\omega}(\sqrt{n} z)\right) \leq\left|G_{\omega}(\sqrt{n} z)\right|<\frac{6 K}{\sqrt{n}}$ and $\left|W_{n}(z)\right|>1$. Therefore, $\Im\left(W_{n}(z)\right)=$ $y-\frac{1}{\sqrt{n}} \Im\left(G_{\omega}(\sqrt{n} z)\right)<\frac{1}{n}+\frac{6 K}{n}$. Hence, we obtain that

$$
\Im\left(F_{\mu_{n}}(z)\right)=y+\frac{\Im\left(W_{n}(z)\right)}{\left|W_{n}(z)\right|^{2}}<\frac{6 K+2}{n}<\frac{C}{n} .
$$

By the previous estimations and (3.1), we conclude that $-\Im\left(G_{\mu_{n}}(z)\right)<\frac{C / n}{(x+1)^{2}}$ for $x \leq-1-\frac{C}{\sqrt{n}}$. It follows that

$$
-\frac{1}{\pi} \int_{A_{1}} \Im G_{\mu_{n}}(z) d x<\frac{1}{\pi} \int_{-\infty}^{-1-\frac{C}{\sqrt{n}}} \frac{C / n}{(x+1)^{2}} d x=\frac{1}{\pi \sqrt{n}} .
$$

By a similar argument, it can be shown that $-\frac{1}{\pi} \int_{A_{2}} \Im G_{\mu_{n}}(z) d x<\frac{1}{\pi \sqrt{n}}$. 
Lemma 3.3. We have that $-\frac{1}{\pi} \int_{\left[-1+\frac{C}{\sqrt{n}}, 1-\frac{C}{\sqrt{n}}\right]} \Im G_{\mu_{n}}(z) d x<\frac{2 C}{3 \sqrt{n}}+\frac{6}{\pi \sqrt{n}}$.

Proof. We deliver the estimation of this integral in three parts.

First, let us suppose that $x \in\left[0.4,1-\frac{C}{\sqrt{n}}\right]$. By (3.3), it follows that $\left|G_{\omega}(\sqrt{n} z)\right|<\frac{30 K}{\sqrt{n}}$.

Our objective is to bound $-\Im\left(G_{\mu_{n}}(z)\right)$. We begin by bounding $\Im\left(F_{\mu_{n}}(z)\right)$. We claim that $\left|W_{n}(z)\right|>0.3$. Indeed, from the definition of $W_{n}(z)$ we see that $\left|W_{n}(z)\right| \geq|z|-\frac{|\alpha|}{\sqrt{n}}-$ $\left|\frac{1}{\sqrt{n}} G_{\omega}(\sqrt{n} z)\right|>x-\frac{|\alpha|}{\sqrt{n}}-\frac{30 K}{n}$. So, the claim follows as $n$ is larger than $20 \cdot 30 K$ and $20^{2} \alpha^{2}$

Next, note that $\Im\left(W_{n}(z)\right)=y-\frac{1}{\sqrt{n}} \Im\left(G_{\omega}(\sqrt{n} z)\right)<\frac{30 K+1}{n}$. It follows that

$$
\Im\left(F_{\mu_{n}}(z)\right)=y+\frac{\Im\left(W_{n}(z)\right)}{\left|W_{n}(z)\right|^{2}}<\frac{1+\frac{1}{0.3^{2}}(30 K+1)}{n}<\frac{C}{n} .
$$

Now, let us bound below $\left|F_{\mu_{n}}(z)\right|$. Observe that

$$
\Re\left(\frac{1}{W_{n}(z)}\right)=\frac{1+\Im\left(W_{n}(z)\right) \Im\left(\frac{1}{W_{n}(z)}\right)}{\Re\left(W_{n}(z)\right)} .
$$

We have that $\Re\left(W_{n}(z)\right) \leq x+\left|\frac{\alpha}{\sqrt{n}}+\frac{1}{\sqrt{n}} G_{\omega}(\sqrt{n} z)\right|<1-\frac{C}{\sqrt{n}}+\frac{|\alpha|}{\sqrt{n}}+\frac{30 K}{n}$. Recall that $\left|W_{n}(z)\right|>0.3$ and $\Im\left(W_{n}(z)\right)<\frac{30 K+1}{n}$. It follows that $1+\Im\left(W_{n}(z)\right) \Im\left(\frac{1}{W_{n}(z)}\right)>1-\frac{(30 K+1)^{2}}{0.3^{2} n^{2}}$. Since the last quantity is bigger than $\Re\left(W_{n}(z)\right)$, as $C>|\alpha|+2$ and $n$ is larger than $(30 k+1)^{2}$ and 16 , then we deduce that $\Re\left(\frac{1}{W_{n}(z)}\right)>1$. Thus,

$$
\left|F_{\mu_{n}}(z)\right| \geq\left|x-\Re\left(\frac{1}{W_{n}(z)}\right)\right| \geq\left|\Re\left(\frac{1}{W_{n}(z)}\right)\right|-x>1-x .
$$

By the above estimations and (3.1), we obtain that $-\Im\left(G_{\mu_{n}}(z)\right)<\frac{C / n}{(x+1)^{2}}$ for $x \in$ $\left(0.4,1-\frac{C}{\sqrt{n}}\right]$. We conclude that

$$
-\frac{1}{\pi} \int_{0.4}^{1-\frac{C}{\sqrt{n}}} \Im G_{\mu_{n}}(z) d x<\frac{1}{\pi} \int_{0.4}^{1-\frac{C}{\sqrt{n}}} \frac{C / n}{(1-x)^{2}} d x<\frac{1}{\pi \sqrt{n}} .
$$

With minor modifications on this argument, we can also deduce that $-\frac{1}{\pi} \int_{\left[-1+\frac{C}{\sqrt{n}},-0.4\right]}$ $\Im G_{\mu_{n}}(z) d x<\frac{1}{\pi \sqrt{n}}$.

Secondly, suppose that $x \in\left(\frac{\sqrt{C}}{\sqrt{n}}, 0.4\right]$. By (3.3), it follows that $\left|G_{\omega}(\sqrt{n} z)\right|<\left(\frac{2}{\sqrt{C}}+\right.$ $\left.\frac{2 \sqrt{n}}{C}\right) K$. Our goal is to bound the expression (3.2).

Note that $\left|W_{n}(z)\right| \leq|z|+\frac{|\alpha|}{\sqrt{n}}+\left|\frac{1}{\sqrt{n}} G_{\omega}(\sqrt{n} z)\right|<x+y+\frac{|\alpha|}{\sqrt{n}}+\frac{2 K}{\sqrt{C n}}+\frac{2 K}{C}<1$ since $C>6 K+1$ and $n$ is larger $20^{2} \alpha^{2}$ and $200 K$. Moreover, as $|z|<\frac{1}{2}$, we obtain that

$$
\left|z W_{n}(z)-1\right| \geq 1-\left|z W_{n}(z)\right|>\frac{1}{2}
$$

Now, we have that $\Im\left(W_{n}(z)\right)=y-\frac{1}{\sqrt{n}} \Im\left(G_{\omega}(\sqrt{n} z)\right)<\frac{1}{n}+\frac{1}{\sqrt{n}}\left(\frac{2}{\sqrt{n} x}+\frac{2}{\sqrt{n} x^{2}}\right) K$, thus

$$
y\left|W_{n}(z)\right|^{2}+\Im\left(W_{n}(z)\right) \leq \frac{2}{n}+\frac{2 K}{n x}+\frac{2 K}{n x^{2}} .
$$

By the above estimations and (3.2), we deduce that

$$
-\frac{1}{\pi} \int_{\frac{\sqrt{C}}{\sqrt{n}}}^{0.4} \Im G_{\mu_{n}}(z) d x<\frac{1}{\pi} \int_{\frac{\sqrt{C}}{\sqrt{n}}}^{0.4} \frac{\frac{2}{n}+\frac{2 K}{n x}+\frac{2 K}{n x^{2}}}{\frac{1}{2}} d x .
$$

Since $\int_{\frac{\sqrt{C}}{\sqrt{n}}}^{0.4} \frac{1}{n x^{2}}<\frac{1}{\sqrt{C} \sqrt{n}}$ and $x>\frac{\sqrt{C}}{\sqrt{n}}$, then we conclude that 
On a Berry-Esseen type limit theorem for Boolean convolution

$$
-\frac{1}{\pi} \int_{\frac{\sqrt{C}}{\sqrt{n}}}^{0.4} \Im G_{\mu_{n}}(z) d x<\frac{2}{\pi n}+\frac{6 K}{\pi \sqrt{C} \sqrt{n}}<\frac{2}{\pi \sqrt{n}} .
$$

By a similar argument we can obtain the same estimation of this integral for $x \in$ $\left[-0.4,-\frac{\sqrt{C}}{\sqrt{n}}\right]$.

Finally, suppose that $x \in\left[-\frac{\sqrt{C}}{\sqrt{n}}, \frac{\sqrt{C}}{\sqrt{n}}\right]$. It follows that $\left|W_{n}(z)\right| \leq|z|+\frac{|\alpha|}{\sqrt{n}}+\left|\frac{1}{\sqrt{n}} G_{\omega}(\sqrt{n} z)\right|$. Moreover, since $\left|G_{\omega}(\sqrt{n} z)\right|<\frac{\omega(\mathbb{R})}{\sqrt{n} y}$, then we conclude that $\left|W_{n}(z)\right|<x+y+\frac{|\alpha|}{\sqrt{n}}+$ $\omega(\mathbb{R})<\frac{\sqrt{C}}{2}$, as $C>4(K+1)^{2}$ and $n$ is larger than $20^{2} \alpha^{2}$ and $16 C^{2}$. Next, note that $\left|z W_{n}(z)\right| \leq|z|\left|W_{n}(z)\right|<\left(\frac{\sqrt{C}}{\sqrt{n}}+\frac{1}{n}\right) \frac{\sqrt{C}}{2}<\frac{1}{2}$ as $n>16 C^{2}$. Thus, we obtain that

$$
\left|z W_{n}(z)-1\right| \geq 1-\left|z W_{n}(z)\right|>\frac{1}{2} .
$$

Now, by the above estimations, we also have that

$$
y\left|W_{n}(z)\right|^{2}+\Im\left(W_{n}(z)\right)<\frac{C}{4 n}+\frac{\sqrt{C}}{2} .
$$

By (3.2), we deduce that $-\Im\left(G_{\mu_{n}}(z)\right)<\frac{C}{2 n}+\sqrt{C}$, and so we conclude that

$$
-\frac{1}{\pi} \int_{-\frac{\sqrt{C}}{\sqrt{n}}}^{\frac{\sqrt{C}}{\sqrt{n}}} \Im\left(G_{\mu_{n}}(z)\right) d x<\frac{2 \sqrt{C}}{\pi \sqrt{n}}\left(\frac{C}{2 n}+\sqrt{C}\right)<\frac{2 C}{3 \sqrt{n}},
$$

as $C>5$ and $n>16 C^{2}$. From this estimation, (3.4), and (3.5), the desired result follows.

Now, we are ready to conclude the proof. Let $\epsilon_{1}=\frac{C}{\sqrt{n}}$ and $\epsilon_{2}=\frac{2}{\sqrt{n}}$. By Theorem 2, we have that

$$
\begin{aligned}
\mu_{n}\left(\left(-\infty,-1-\epsilon_{1}-\epsilon_{2}\right]\right) & \leq-\frac{1}{\pi} \int_{-\infty}^{-1-\epsilon_{1}} \Im\left(G_{\mu_{n}}(z)\right) d x+\frac{2 y}{\pi \epsilon_{2}}, \\
\mu_{n}\left(\left[-1+\epsilon_{1}+\epsilon_{2}, 1-\epsilon_{1}-\epsilon_{2}\right]\right) & \leq-\frac{1}{\pi} \int_{-1+\epsilon_{1}}^{1-\epsilon_{1}} \Im\left(G_{\mu_{n}}(z)\right) d x+\frac{2 y}{\pi \epsilon_{2}}, \text { and } \\
\mu_{n}\left(\left[1+\epsilon_{1}+\epsilon_{2}, \infty\right]\right) & \leq-\frac{1}{\pi} \int_{1+\epsilon_{1}}^{\infty} \Im\left(G_{\mu_{n}}(z)\right) d x+\frac{2 y}{\pi \epsilon_{2}} .
\end{aligned}
$$

The previous lemmas implies that $\mu_{n}\left(\left(-\infty,-1-\epsilon_{1}-\epsilon_{2}\right]\right)<\frac{1}{\pi \sqrt{n}}+\frac{1}{\pi \sqrt{n}}, \mu_{n}([-1+$ $\left.\left.\epsilon_{1}+\epsilon_{2}, 1-\epsilon_{1}-\epsilon_{2}\right]\right)<\frac{2 C}{3 \sqrt{n}}+\frac{6}{\pi \sqrt{n}}+\frac{1}{\pi \sqrt{n}}$, and $\mu_{n}\left(\left[1+\epsilon_{1}+\epsilon_{2}, \infty\right]\right)<\frac{1}{\pi \sqrt{n}}+\frac{1}{\pi \sqrt{n}}$. Since $\frac{2 C}{3 \sqrt{n}}+\frac{11}{\pi \sqrt{n}}<\frac{C}{\sqrt{n}}+\frac{2}{\sqrt{n}}=\epsilon_{1}+\epsilon_{2}$ for $C>5$, then we obtain that

$$
\mu_{n}\left(\left(-1-\epsilon_{1}-\epsilon_{2},-1+\epsilon_{1}+\epsilon_{2}\right) \cup\left(1-\epsilon_{1}-\epsilon_{2}, 1+\epsilon_{1}+\epsilon_{2}\right)\right)>1-\epsilon_{1}-\epsilon_{2} .
$$

Therefore, by Proposition (2.1), we conclude that

$$
d_{l e v}\left(\mu_{n}, \mathbf{b}\right) \leq \frac{7}{2} \frac{C+2}{\sqrt{n}} .
$$

The author thinks that it is an interesting question if for finite fourth moment, or even finite absolute third moment, we still have an order of convergence $O\left(n^{-1 / 2}\right)$ in Theorem 1. 
On a Berry-Esseen type limit theorem for Boolean convolution

\section{References}

[1] O. Arizmendi \& M. Salazar, A Berry-Esseen type limit theorem for Boolean convolution, Archiv der Mathematik 111.1 (2018), 101-111. MR3816982

[2] O. Arizmendi, M. Salazar \& J.C. Wang, Berry-Esseen Type Estimate and Return Sequence for Parabolic Iteration in the Upper Half-Plane, International Mathematics Research Notices (2019). MR4349228

[3] Z. D. Bai, Convergence rate of expected spectral distributions of large random matrices part i: Wigner matrices, Advances In Statistics (2008), 60-83. MR3948975

[4] A. C. Berry, The accuracy of the Gaussian approximation to the sum of independent variates, Transactions of the american mathematical society 49.1 (1941), 122-136. MR0003498

[5] G. P. Chistyakov \& F. Götze, Limit theorems in free probability theory. I, The Annals of Probability 36.1 (2008), 54-90. MR2370598

[6] C.G. Esseen, On the Liapunoff limit of error in the theory of probability, Arkiv for matematik astronomi och fysik 28 (1942), 1-19. MR0011909

[7] W. Feller, An Introduction to Probability Theory and Its Applications 2, 2nd ed. Wiley, New York, 1971. MR0270403

[8] A. L. Gibbs \& F. E. Su, On choosing and bounding probability metrics, International statistical review 70.3 (2002), 419-435.

[9] T. Hasebe, On monotone convolution and monotone infinite divisivility, (2010), preprint, arXiv:1002.3430. MR3010792

[10] N. Muraki, The five independences as natural products, Infinite Dimensional Analysis, Quantum Probability and Related Topics 6.03 (2003), 337-371. MR2016316

[11] R. Speicher \& R. Woroudi, Boolean convolution, Fields Inst. Commun. 12 (1993), 267-279. MR1426845

[12] G. Teschl, Jacobi operators and completely integrable nonlinear lattices, Mathematical Surveys and Monographs 72, American Mathematical Society, 2000. MR1711536

Acknowledgments. The author appreciates the various suggestions and comments from the referees which helped improve the presentation of this paper. The author is also indebted to Octavio Arizmendi for many stimulating discussions. 